EESTI NSV TEADUSTE AKADEEMIA TOIMETISED 1952. I k., nr. 1 ИЗВЕСТИЯ АКАДЕМИИ НАУК ЭСТОНСКОИ ССР 1952. Том I, № 1

\title{
БОРЬБА МАТЕРИАЛИЗМА И ИДЕАЛИЗМА В СОВРЕМЕННОЙ КОсмолОГИи *
}

\author{
Г. И. НАAН, \\ действительный член Академии Наук Эстонской ССР
}

Қосмология является, как известно, частью астрономии и занимается проблемами строения вселенной. Она стремится установить законы распіределения, движения и развития материи в возможно больших областях пространства-времени. Она опирается на данные наблюдательной астрономии и обобщает их с помощью физических теорий. Поскольку область вселенной, доступная нашим наблюдениям, с развитием средств и методов наблюдения беспрестанно расширяется, а космология идет, так сказать, по пятам за наблюдательной астрономией, то космологии неизбежно приходится иметь дело с недостаточно еще изученным материалом. Мало того. Космология всегда выходит за пределы того, что доступно наблюдениям на данном этапе развития науки, стремясь экстраполировать закономерности распределения, движения и развития материи (космических объектов) в данной, доступной области вселенной, на бо́льшие, пока еще недоступные ее области. Отсюда ясно, что роль правильной методологии, философии, которая велика в развитии любой отрасли естествознания, в развитии космологии исключительно велика. В наши дни научная космология может иметь теоретической основой своего развития только принципы диалектического материализма. Причины глубокого кризиса космологии в капиталистических странах лежат как раз в том, что космологию пытаются строить на идеалистической и метафизической «основе». Ясно, в частности, - это вытекает из самого характера данных, с которыми имеет дело космология, - что всякая попытка игнорировать учение диалектического материализма о соотношении абсолютной и относительной истины должна в области космологических обобщений жестоко мстить за себя. Опыт буржуазной космологии подтверждает это положение.

Но это лишь одна сторона тесной связи космологии с философией. Эта связь имеет и другую сторону. В силу самого характера космологии как науки ее выводы и обобщения имеют большое значение для философии. Наиболее общие ее выводы входят в философию даже в качестве собственных ее положений. Естественно поэтому, что космология является ареной жестокой борьбы философских партий - материализма и идеализма, борьбы, которая является вместе с тем борьбой науки и религии, борьбы, за которой стоит борьба политинеских партий и общественных классов. Центральным вопросом борьбы является вопрос о конечности или беско-

\footnotetext{
* Доклад на сессии Академии Наук Эстонской ССР 17 июня 1950 г.
} 
нечности мира. Линия диалектического материализма отстаивает бесконечность вселенной в пространстве и времени. Противоположная, идеалистически-поповская линия защищает антинаучное представление о конечности мира в пространстве и времени, поворачивая науку вспять, к обветшалым птоломеевско-схоластическим воззрениям.

Астрономия, как указывает И. В. Сталин, стала, собственно, наукой только со времени создания Коперником гелиоцентрической космологической системы.

Существенной чертой господствовавшей до этого геоцентрической космологической системы Аристотеля-Птоломея, кроме представления о центральном положении Земли, как центра тяготения для всех тел вселенной, является представление о пространственной конечности мира. В этой системе в центре вселенной находится Земля, вокруг которой обращаются Луна, Меркурий, Венера, Солнце, Марс, Юпитер, Сатурн и сфера неподвижных звезд. Эта последняя сфера, по Аристотелю, является границей материальной вселенной, за которой находится сфера нематернального «первого двигателя», приводящего в движение все материальные сферы. Эта концепция была принята схоластами и стала существенной частью официального церковного мировоззрения средневековья. В частности, представление о пространственной конечности видимого мира, при бесконечности мира духовного, служило в руках церкви аргументом в пользу первичности духа и вторичности жнизменной» видимой материи.

С гелиоцентрической системой почти неразрывно связано представление о бесконечности вселенной. Еще античный предшественник Коперника Аристарх Самосский, высказывавший догадку об обращении Земли и планет вокруг Солнца, учил о бесконечности вселенной. Что касается самого Коперника, то он, правда, придерживался на этот счет еще взглядов Птоломея и Аристотеля, согласно которым все неподвижные звезды находятся на одном небесном «круге». Но это был явный пережиток старых взглядов. Уже Джордано Бруно сделал естественно вытекающий из системы Коперника вывод о бесконечности вселенной. Труды Галилея, Кеплера и Ньютона принесли полное торжество системе Коперника. C тех пор в науке господствовал (можно сказать: безраздельно господствовал) гелиоцентризм и положение о бесконечности вселенной.

Дело приняло, однако, иной оборот в эпоху империализма. Империализм, по гениальной характеристике В. И. Ленина, означает «реакцию по всей линии». Означает он реакцию и по линии развития науки, в частности, естествознания. Одним из характерных проявлений этой реакции являются попытки так или иначе возродить обветшалую космологическую систему Птоломея. Здесь мы имеем дело не только с попытками приписать вселенной пространственно замкнутый характер, но и со стремлением возродить птоломееву систему в ее собственно геоцентрической части, то есть, с утверждениями, что «все равно», считать ли, что Земля обращается вокруг Солнца, или что Солнце обращается вокруг Земли; обе системы являіотся-де равно допустимыми описаниями фактов.

Остановимся сперва на попытках второго рода. Основаны они на неправильной интерпретации общей теории относительности, созданной Эйнштейном в 1916 году. Согласно этой интерпретации, с точки зрения обцей теории относительности все системы отсчета, в том числе и движущиеся ускоренно, совершенно равноправны - равноправны не только в кинематическом отношении, но и в физическом отношении, вообще равноправны. Если это так, то, конечно, безразлично, считать ли, что Земля обращается вокруг Солнца, или что Солнце обращается вокруг Земли; считать ли, что вращающийся волчок действительно вращается относительно окружающих его тел, или, наоборот, считать, что когда мы двумя 
пальцами запускаем волчок, то волчок остается в покое, а вся вселенная вокруг него приходит в движение. С этой точки зрения борьба Коперника, Бруно, Іалилея против схоластики и церковного мракобесия была бессмысленной. К примеру, американский неопозитивист Ганс Рейхенбах $\left({ }^{18}\right)$ пишет: «Нет смысла говорить о различии в истинности между концепциями Коперника и Птоломея: обе концепции суть равно допустимые описания. Истинность того, что рассматривалось как победа западной мудрости, противостоящей мудрости античной; является сомнительной». Следует отметить, что сам Эйнштейн также придерживается неправильного взгляда на созданную им теорию. В написанной совместно с Инфельдом книге $\left({ }^{14}\right)$ он пишет следующее: «Можем ли мы сформулировать физические законы таким образом, чтобы они были справедливы для всех систем координат, не только для систем, движущихся прямолинейно и равномерно, но и для систем, движущихся совершенно произвольно по отношению друг к другу?.. Борьба между воззрениями Птоломея и Коперника, столь жестокая в ранние дни науки, стала бы тогда совершенно бессмысленной ... Два предложения - «Солнце покоится, а Земля движется» и- «Солнце движется, а Земля покоится» - означали бы просто два различных соглашения о двух различных системах координат ... Это, в самом деле, оказывается возможным!»

Әти утверждения совершенно неосновательны. Қак показал академик В. А. Фок $\left({ }^{7-9}\right)$, все соображения в пользу относительности ускорения в обшей теории относительности могут быть легко опровергнуты.

Первым соображением в пользу относительности ускорения является прннцип эквивалентности ускорения и тяготения. Но принцип этот применим, строго говоря, только к бесконечно малым областям пространства, где гравитационное поле однородно. Практически требуется, чтобы область пространства была настолько мала, чтобы пространственно-временными изменениями поля в пределах этой области можно было пренебречь. К системам, подобным солнечной системе, это неприменимо, ибо в пределах солнечной системы поле никак не может считаться однородным.

Другим аргументом в пользу указанного выше истолкования общей тєории относительности является ссылка на невозможность в общей теорин относительности ввести (в общем случае) декартову систему координат. В силу неевклидовости пространства мы вынуждены в общей теории относительности применять не наглядные координатные параметры гауссовы координаты. Это как будто говорит в пользу невозможности в рамках общей теории относительности представления о привилегированных (инерциальных) системах отсчета. Однако, как показал В. А. Фок ( ${ }^{7}$ ) в 1939 году, и в общей теории относительности можно ввести величины вполне аналогичные прямоугольным декартовым координатам и времени в частной теории относительности - гармонические координаты.

Существенным условием для этого является требование, чтобы на достаточном удалении от всех масс рассматриваемой системы («на бесконечности») метрика пространства-времени была бы псевдо-евклидовой. Для астрономических систем, таких как солнечная система, это требование выполняется. Поэтому система отсчета, связанная с Солнцем, и в общей теории относительности является привилегированной. Вводится она для всей области пространства, достаточно удаленной от ближайших звезд, и может, в сущности, рассматриваться как вводимая однозначно * инерциальная система отсчета. Все движения тел солнечной системы следует относить именно к этой системе отсчета, а не к произвольным системам отсчета, связанным, например, с планетами (в частности, с Землей).

\footnotetext{
* Однозначно с точностью до преобразования Лоренца.
} 
Нам кажется, что это последнее обстоятельство заслуживает пристального внимания. В. А. Фок справедливо указывает на необоснованность претензий Әйнштейна и его сторонников на универсальную применимость общей теории относительности, в частности, на применимость ее к решению проблемы элементарных частиц. Общая теория. относительности есть теория тяготения и применяться она должна к тем явлениям, в которых тяготение играет преобладающую роль, то есть прежде всего к явлениям астрономического масштаба. Әто соображение В. А. Фока, на наш взгляд, можно дополнить другим, пожалуй, не менее важным соображением. И в применении общей теории относительности к астрономическим проблемам для Әйнштейна и вообе буржуазной теоретической физнки характерны крайняя абстрактность, отрыв от действительности рассмотрение проблем тяготения «вообще», стремление получить решения, справедливые всегда и всюду, игнорирование данных о реальном распределении материи в пространстве. При попытках решения космологической проблемы предположение о хаотическом, в среднем равномерном распределении материи выступает как специальный (притом существенный) постулат. Между тем данные наблюдательной астрономии. говорят в піользу того, что космические объекты распределены не беспорядочно, а образуют входяшие друг в друга системы (об этом подробнее ниже). Вследствие этого метрика пространства-времени на достаточном удалении от тел каждой из таких систем является с достаточной степенью точности псевдо-евклидовой, и можно предположить, что возможность введения инерциальной системы отсчета однозначно для пространства каждой из снстем является общей закономерностью, обусловленной реальным распределением материи. Если это так, то замечание В. А. Фока, что рассмотренная им «физическая задача не имеет никакого отношения к т. н. космологической проблеме» (стр. 408 назв. работы), требует коррективов. Можно утверждать, что полученные В. А. Фоком результаты являются серьезґейшим аргументом против предлагаемого Әйнштейном и его сторонниками решения космологической проблемы.

Третьим доводом в пользу относительности ускорения является ссылка на ковариантность уравнений общей теории относительности относительно «пр извольных» преобразований координат. Этот аргумент также может быть легко опровергнут. В. А. Фок $\left({ }^{9}\right)$ справедливо указывает на то обстоятельство, что ковариантность законов физики не составляет монополии общей теории относительности: она свойственна всякой правильной. физической теории. В частности, прекрасно разработан математический аппарат (уравнения Лагранжа второго рода), позволяющий формулировать законы классической механики ковариантным образом. Между тем ускорение в классической механике сохраняет физически абсолютный характер. К этому мы, пожалуй, добавили бы еще следующее. Принцип ковариантности сам по себе говорит только о математической формулировке законов. Физическое содержание в общей теории относительности он получает только через принцип эквивалентности. ПI. Г. Бергман $\left({ }^{3}\right)$ в своей книге, просмотренной и одобренной Әйнштейном, рассматривает поэтому принцип общей ковариантности, как просто математическое выражение принципа эквивалентности. Принцип же эквивалентности имеет лишь локальный характер.

Отсюда видно, что попытки возродить обветшалую систему Птоломея, как якобы равноправную с системой Коперника, несостоятельны.

С философской точки зрения они означают попытку дать естественнонаучное подкрепление точке зрения, которая была выдвинута еще монахом Осиандером в предисловии к первому изданию книги Коперника $\left({ }^{15}\right)$, н возведена в особый философский принцип махистами: научные теории 
яеляются не отражением объективной реальности, а лишь более или менее удобной фикцией, необходимой для вычислений, но не содержащей ничего достоверного.

Физической основой этих попыток является ошибочное представление об относительности ускорения в общей теории относительности, возникшее на базе абстрактной и формальной интерпретации этой физической теории.

Та же махистская философия, тот же формализм и представление об относительности ускорения проходят красной нитью и через «доказательства» пространственной конечности мира, «основанные» на общей теории относительности.

Эйнштейн $\left({ }^{13}\right)$ выдвигает в пользу пространственной конечности вселенной следующие доводы. Во-первых, «с точки зрения теории относительности условия пространственной замкнутости много проще, чем пограничные условия для бесконечности». Но этот довод имеет какую-то силу только с точки зрения махистской «экономии мышления». С точки зрения марксистской философии он вовсе не доказывает еще его истинности. Математические затруднения общей теории относительности с граничными условиями - не резон для отказа от научного взгляда на мир в пользу идеалистически-поповского. Здесь уместно привести слова Энгельса $\left({ }^{1}\right)$ : «Идеальная потребность математика вовсе не есть принудительный закон для реального мира».

Известно, что классическая (ньютоновская) теория гравитации при ее распространении на большие участки вселенной наталкивается на серьезное затруднение: при равномерном распределении звезд притяжение, испытываемое каждой из них со стороны всех остальных, должно быть бесконечно большим, чего в -действительности не наблюдается (парадокс Неймана-Зелигера). Аналогичное затруднение имеет место и в релятивистской теории тяготения. Буржуазная космология и предлагает «разрешить» это затруднение «просто» - отказаться от самой идеи бесконечности вселенной. Однако это «решение» отнюдь не является необходимым. Еще Ламберт в XVIII веке и Шарлье в нашем веке выдвинули идею «иерархического» строения бесконечной вселенной, дающую возможность разрешить гравитационный и аналогичный ему фотометрический парадокс. Материя предполагается распределенной не равномерно, а группирующейся в бесконечное множество входящих друг в друга космических систем возрастающего порядка сложности. $\mathrm{N}_{1}$ звезд образуют звездную систему первого порядка, $\mathrm{N}_{2}$ звездных систем первого порядка образуют звездную систему второго порядка, и т. д.

Развитие идеи об иерархическом строении бесконечной вселенной на основе современных данных проводится советскими учеными. В первую очередь здесь нужно отметить акад. В. Г. Фесенкова $\left({ }^{6}\right)$ и проф. М. С. Эйгенсона $\left({ }^{10-12}\right)$. М. С. Эйгенсон показал, что гравитационный парадокс устраняется при предположении о структурности вселенной в духе Ламберта. Необходимым условием устойчивости иерархической лестницы является физическая автономность систем, состоящая в том, что движение частицы внутри каждой системы определяется создаваемым этой системой полем и не зависит существенно от полей систем более высоких порядков. Для этого нужно, чтобы размеры тел (систем) были много меньше расстояний между ними, или, что равносильно, чтобы средняя плотность системы $(\mathrm{i}+1)$-го порядка была много меньше средней плотности системы і-го порядка. В доступной нам сейчас части вселенной это условие выполняется (средняя плотность солнечной системы имеет порядок величины $10^{-12}$, Галактики $10^{-25}$, известной части Метагалактики $\left.10^{-30} \mathrm{r} / \mathrm{cm}^{3}\right)$. Это обстоятельство, на наш взгляд, может быть поставлено в связь с указанными выше результатами, полученными В. А. Фоком. 
Вследствие монотонного убывания средней плотности космических систем пространство на достаточном удалении от всех тел данной системы с достаточной степенью точности является евклидовым. Отсюда можно сделать вывод о возможности введения инерциальной системы отсчета для пространства каждой данной космической системы. Следует ясно представлять себе, что «граничные условия для бесконечности» суть граничные условия для бесконечности в физическом смысле, то есть для достаточного удаления от тел данной космической системы. Для всей вселенной сама постановка вопроса абсурдна; в ней смешивается бесконечность в физическом смысле с математической бесконечностью.

Вторым доводом в пользу конечности мира у Әйнштейна является ссылка ніа так наз. принцип Маха. Әтот «принцип» Әйнштейн формулирует следующим образом: «материальная точка должна двигаться без ускорения не по отношению к пространству, а по отношению к центру остальных масс мира». Обосновывается это «гносеологическими соображениями», а именно тем, что причина инерциального движения должна быть «наблюдаемым опытным фактом», привилегированная же система отсчета есть якобы фикция, а не наблюдаємый факт. Внешне принцип и его обоснование выглядят весьма научно и исходят из опыта. Но это только внешне. Здесь мы имеем, на наш взгляд, дело с обычной маскировкой идеализма под жопыт», столь характерной для махизма, когда объективная реальность объявляется ненаблюдаемой, а всевозможные домыслы возводятся в ранг наблюдательного факта. В действительности инерциальная система отсчета не является какой-то фикцией; выбор ее диктуется реальным и, конечно, вполне наблюдаемым распределением масс. Что же касается самого принципа Маха, то он просто-напросто постулирует конечность мира, ведь только конечная система может иметь центр инерции. Бессмысленно прилагать это понятие к бесконечной системе. Бесконечность вселенной необходимо означает отсутствие у нее центра к каком бы то ни было смысле, в том числе и центра инерции. Таким образом, доводом в пользу конечности мира объявляется принцип Маха, а принцип Маха исходит из конечности мира!

В-третьих, по утверждению Эйнштейна, бесконечная вселенная возможна (с точки зрения общей теории относительности) только при исчезающе малой средней плотности материи в ней, что, дескать, мало вероятно. Әтот аргумент несерьезен. Уже в Галактике средняя плотность имеет порядок величины $10^{-25} \mathrm{r} / \mathrm{cm}^{3}$, то есть практически она исчезающе мала. Высшим ступеням структурной лестницы должна соответствовать еще меньшая плотность.

Таким образом, все доводы Эйнштейна неосновательны.

Следует, наконец, остановиться еще на одном обстоятельстве. Авторы «теорий» конечности мира молчаливо исходят из того, что гравитационные уравнения в эйнштейновской форме являются некоей абсолютной истиной в конечной инстанции. Только при этом условии их можно распространять на всю вселенную в целом. Но ясно, что это совершенно беспочвенная претензия. Релятивистская теория тяготения применима к более сильным гравитационным полям, чем ньютоновская, но это вовсе не значит, что она применима к сколь угодно сильным и сколь угодно обширным гравитационным полям. Как и всякая (правильная) естественно-научная теория она представляет истину относительную. А. Ф. Богородский $\left({ }^{45}\right)$ привел веские соображения в пользу того, что эйнштейновская форма уравнений является не единственно возможной в релятивистской теории тяготения и что ее следует скорее рассматривать как лишь первое приближение (считая ньютоновскую теорию нулевым гіриближением) в решении проблемы. Поэтому эти уравнения незаконно 
применять к столь обширным областям мира, как система галактик, и, конечно, не может быть и речи о применении их ко всей вселенной в целом.

Так обстоит дело с попытками доказать конечность вселенной в пространстве. Они совершенно несостоятельны. Что же касается попыток доказать ограниченность вселенной во времени, то они просто вздорны.

В качестве наиболее веского довода в пользу того, что мир существовал не вечно, что он был сотворен несколько миллиардов лет тому назад, буржуазные космологи выдвигают «красное смещение» - тот факт, что в спектрах удаленных галактик линии смещены к «красному» концу спектра, то есть в сторону длинных волн. Интерпретация этого явления представляет трудности. Наиболее вероятным на сегодня кажется истолкование его как допплер-эффекта, то есть как результата удаления галактик друг от друга. Известно также, что существует приблизительно линейная зависимость между величиной смещения (согласно допплеровской интерпретации - скоростью удаления) и расстоянием до галактики. Буржуазные космологи утверждают, что красное смещение является доказательством расширения вселенной и что линейный характер зависимости между скоростью и расстоянием дает возможность вычислить ее возраст. Зная скорость удаления и предполагая, что она зависела и зависит от расстояния строго линейно, можно подсчитать, когда (в прошлом) талактики должны были находиться «в одной точке». Получаются цифры порядка полутора-двух миллиардов лет. Эти полтора-два миллиарда лет рассматриваются как «возраст вселенной», как время, истекшее с момента ее сотворения. Правда, многие буржуазные астрофизики стыдятся называть вещи своими именами и предпочитают говорить об «особой точке» и т. п. Но ряд других считает это излишней щепетильностью. Например, президент Әдинбургского Королевского общества (Шотландской. академии наук) проф. Э. Т. Уайтеккер $\left({ }^{19}\right)$ пишет черным по белому, что «логичнее всего постулировать сотворение мира из ничего актом божественной воли». Проф. Э. А. Милн $\left({ }^{17}\right)$ (упомянутый А. А. Ждановым в выступлении на философской дискуссии 1947 года), также заявляет, что здесь мы можем «проследить средствами физики перст божий».

Бросается в глаза предвзятость всех этих построений, имеющих единственную цель - услужить поповщине. Расширение (если оно действительно имеет место, что нельзя считать окончательно доказанным) может охватывать огромные области пространства, которые, тем не менее, составляют лишь крохотную частицу бесконечной вселенной. Рядом с участками, где пронсходит расширение, могут существовать миллионы участков, где происходит сжатие или имеет место относительное равновесие галактик. Буржуазные же космологи без всяких сснований считают, что расширение охватывает всю вселенную в целом. Далее. Расширение, происходящее на данном участке в течение, скажем, ста миллионов лет, может затем смениться состоянием относительной устойчивости или сжатием и т. д. Весь опыт естествознания говорит о том, что формы движения материи бесконечно разнообразны, что происходит непрестанное превращение одних форм в другие. Буржуазные же космологи экстраполируют расширение на миллиарды лет в прошлое. И уж совершенно смехотворна в качестве «возраста вселенной» сама цифра в полтора-два миллиарда лет. Возраст земной коры достаточно надежно оценивается примерно в два-три миллиарда лет. Земля, как астрономическое тело, конечно, старше. Звезды еще старше. Звездные системы, в свою очередь, еще старше.

Некоторые буржуазные астрофизики пытаются спасти положение идеа- 
лизма тем, что объявляют этот возраст отдельных космических объектов,

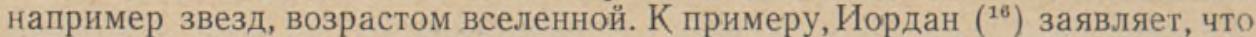
«не только атомы, звезды и системы млечных путей, но также и престранство и время» были сотворены «десять миллиардов лет тому назад». Названный выше Уайттекер $\left({ }^{19}\right)$ ссылаясь на то, что возраст типичной звезды едва ли превышает $10^{10}$ или $10^{11}$ лет, что двойные звезды должны распадаться примерно в $5.10^{9}$, а рассеянные звездные скопления типа Плеяд в $3.10^{\circ}$ лет, делает вывод, что «должна была быть эпоха около $10^{\circ}$ или $10^{10}$ лет тому назад, за которой космос, если он вообще существовал, существовал в форме, совершенно отличной от всего того, что известно нам... Мы можем, пожалуй, без опаски считать это сотворением мира». В этих смехотворных по своей наивности, но характерных для находящейся в состоянии маразма современной буржуазной науки, «подсчетах» предполагается, что все объекты во вселенной были сотворены өдновременно, что после этого мир может только «стареть», идя навстречу своей гибели. Между тем работы советских астрономов показывают, что во вселенной имеются объекты самых различных возрастов: от звезд типа Вольф-Райе и ядер планетарных туманностей (менее $10^{6}$ лет) и некоторых «расточительных» сверхгигантов $\left(10^{6}-10^{7}\right.$ лет) до молодых $\left(10^{\circ}-10^{10}\right.$ лет) и старых $\left(10^{11}-10^{12}\right.$ лет) звезд. Возраст Галактики вряд ли может быть менее $10^{13}$ лет. В частности, работы В. А. Амбарцумяна $\left(^{2}\right)$ и его сотрудников о звездных ассоциациях, выполненные в последние годы, убедительно доказывают наличие и сравнительное обилие в Галактике молодых объектов.

Все эти данные говорят о том, что процесс образования звезд и других космических объектов идет непрерывно, идет и в настоящее время.

Эти данные наносят сокрушительный удар идеалистическим концепциям. Эти данные противоречат предвзятым и антинаучным представлениям о сотворении мира и его односторонней регрессивной эволюции, но находятся в полном согласии с положением диалектического материализма о вечности вселенной, о несотворимости и неуничтожимости материи и движения. Возраст космических объектов - это не время, истекшее с момента их мифического «сотворения», а время пребывания материи в данном качественном состоянии. До этого она, естественно, могла пройти бесконечный ряд других состояний, также как пройдет бесконечный ряд состояний после этого.

Қак известно, возраст отдельного человека редко превышает сто лет. Однако никто не станет утверждать на основании этого, что человечество существует лишь сто лет, что сто лет существуют органическая жизнь на Земле, сама Земля и вся вселенная. Между тем «подсчет» «возраста» вселенной буржуазными космологами проводится именно этим методом, с той только разницей, что вместо возраста отдельного человека берется возраст отдельного космичческого объекта.

В современной космологии, как и в других отраслях естествознания, мы видим ожесточенную борьбу материализма и идеализма. Буржуазная космология, руководствуясь «принципами» идеализма и метафизики, пришла к состоянию маразма и разложения, сомкнулась непосредственно с оголтелой поповщиной. Космологические и космогонические работы советских ученых, имеющие своей теоретической основой принципы диалектического материализма, показывают действительные пути развития науки о строении вселенной.

Несмотря на отчаянные потуги, буржуазные космологи не смогли (и, конечно, не смогут) привести ни одного хоть сколько-нибудь веского факта или соображения, которые заставили бы нас усомниться в истинности 
научного материалистического положения о бесконечности вселенной в: пространстве и времени в пользу идеалистически-поповского представления о конечности вселенной.

\section{ЛИТЕРАТУРА}

1. Ф. Э н г е л с с, Анти-Дюринг, 1950, стр. 49 .

2. В. А. А м б а р ц у м я н, Эволюция звезд и астрофизика, Ереван, 194 I.

3. П. Г. Б е р г м а н, Введение в теорию относительности, М., 1948.

4. А. Ф. Бо го р од с к и й, О гравитационных уравнениях теории относительности, Циркуляр Главн, астр. обсерватории, № 32, 1941.

5. А. Ф. Б о г о ро д с к и й, Космологинеская проблема, Публикаціі Киивьскоі астрономичноі обсерваторіi, № $1,1946$.

6. В. Г. Ф е с ен ков, О значении космической рассеянной материи в явлении светимости ночного неба и в связи с вопросом о бесконечности вселенной, Астрон. журн., т. 14, 1937.

7. В. А. Ф о к, О движении конечных масс в общей теории относительности, Журн. экспер. и теор. физики, т. 9, 1939.

8. В. А. Фок, Об интегралах движения центра инерции двух конечн. масс в общей теории относительности, Доклады АН СССР, т. 32, 1941.

9. В. А. Ф о к, Система Коперника и система Птоломея в свете общей теории относительности, Сборн. «Николай Коперник», М.-Л., 1947.

10. М. С. Э й ге н сон, Поверхностная яркость ночного неба и бесконечность астрономической вселенной, Астрон. журн., т. 16, 1939.

11. М. С. Э й г е н с о н, Вселенная Ламберта и парадокс Зелигера, Доклады АН СССР, T. $26,1940$.

12. М. С. Э й г е н сон, Космологическая относительность и релятивистская космология, Доклады АН СССР, т. 26, 1940.

13. А. Э й н ш т е й н, Основы теории относительности, М.-Л., 1935.

14. А. Э й н ш т е й н и Л. Ин ф е ль д, Эволюция физики, М.-Л., 1948.

15. N. Copernicus, De Revolutionibus Orbium Coelestium, Norimberg, 1543.

16. P. Jordan, Physies of the 20th Century, New-York, 1944.

17. E. A. M illn e, Relativity, Gravitation and World-Structure, Oxford, 1935.

18. H. Reichen b a ch, From Copernicus to Einstein, New-York, 1942.

19. E. T. Whittaker, The Beginning and End of the World, London, 1943.

20. E. T. Whittaker, Space and Spirit, London, 1946. 